\title{
Vigilance performance as related to task instructions, coaction, and knowledge of results
}

\author{
JAMES M. HUNTERMARK and KENNETH L. WITTE \\ University of Arkansas, Fayetteville, Arkansas 72701
}

\begin{abstract}
The vigilance of college students (32 men, 32 women) as related to three variables was assessed. Observers given evaluative-test instructions and knowledge of results or tested with the experimenter present detected more signals than their respective counterparts given standard-task instructions and no knowledge of results or tested with the experimenter absent. Overall, a vigilance decrement occurred across the 48-min watch. Of the three independent variables, only evaluative instructions was effective in preventing a decrement.
\end{abstract}

In laboratory vigilance tasks, human observers monitor monotonous displays for brief and nonpersistent signals over extended periods of time. A recurrent finding is a vigilance decrement; that is, accuracy of signal detection deteriorates with time. However, not all studies have reported vigilance decrements. Decrements are not as common in studies involving military and other "real-life" situations as they are in laboratory studies. Apparently, an important variable in this regard is the observer's level of motivation (Mackworth, 1970; Smith \& Lucaccini, 1969). The present study examined the possible motivational effects of three variables: instructions, coaction (presence of another), and knowledge of results (KR).

The first variable, instructions, was manipulated by giving observers either evaluative-test or standard-task instructions. Research in other areas has indicated that instructions of the former type have a beneficial effect upon performance, for example, paired associate learning (Caron \& Caron, 1965) and short-term memory (Witte \& Huntermark, 1975). Task instructions have been a neglected variable in the vigilance research, and the results of the available research are inconclusive at this time. For example, Lucaccini, Freedy, and Lyman (1968) compared the behavior of observers told that the vigil was either a "challenge" or "monotonous." The number of detections was greater for the former than for the latter group, and a vigilance decrement was not obtained for either group. Tolin (1970), using the same instructions as Lucaccini et al., found no differences between the two instruction groups, and a reliable vigilance decrement was obtained. Of the many procedural differences between these two studies, perhaps the one primarily responsible for the conflicting results, and the second variable of present interest, is

This research was the basis for a doctoral dissertation by the first author under the direction of the second author. Reprint requests should be sent to the first author, who is now at Livingston University, College of General Studies, Division of History and Social Sciences, Livingston, Alabama 35470. coaction. In the Lucaccini et al. study, the observers were monitored in the presence of the experimenter, while this was not the case in the Tolin study. It is possible that observers feel "challenged" or tested only if someone is present to monitor their behavior.

The available vigilance research concerned with coaction effects indicates that the status of the other person is important. Apparently, if coaction involves the presence of someone of equal status as the observer (the use of multiple monitors), then coaction effects are not obtained (e.g., Bergum \& Lehr, 1962). However, coaction effects do seem to result from the presence of the experimenter, or some other authority figure, who either directly or indirectly leads the observer to believe his behavior is being evaluated (Bergum \& Lehr, 1963).

A number of vigilance studies have examined the effects of knowledge of results. Studies simply concerned with the effects of KR vs. no KR (McCormick, 1959; Wiener, 1963), as well as studies comparing the effects of variations in the amount (frequency) of KR (Johnson \& Payne, 1966; McCormick, Binding, \& McElerhan, 1963), indicate that KR facilitates vigilance. Research from a third area, that concerned with the effects of true vs. false KR, indicates that the positive effect of $K R$ is due to a motivational rather than an informational variable. If the effects of $K R$ are informational, then the vigilance of observers given false KR should not be as accurate as that of observers given true KR. However, the vigilance of these two groups does not differ, and both differ from observers not given KR (e.g., Antonelli \& Karras, 1967). For a more complete review of the research concerned with the effects of KR, as well as the other two variables of concern, on vigilance, see Huntermark (1977).

In addition to the individual effects of the three variables under investigation, it is possible that they interact with each other. To date, with the apparent exception of one study that examines the joint effects of coaction and KR (Hardesty, Trumbo, \& Bevan, 1963), no study has included any two of these variables within the same experimental design. As indicated 
previously, the motivational effects of instructions and coaction are mediated by the threat of potential evaluation. Hardesty et al. (1963) posited that the motivational effects of KR involve the same process. It might be intuited that the motivational increments resulting from these three sources simply summate, producing increasingly higher levels of signal detection. Or, it may be the case that once observers are sufficiently motivated, signal detection is maximal. Or still yet, it may be the case that there is an optimal level of motivation for accurate signal detection, and if the observer's motivation is either too low or too high, then vigilance decreases.

As Borgartz (1976) has cogently demonstrated, intuition is inadequate as a careful guide to the prediction and interpretation of interactions. Given the issues raised by Bogartz (1976; Bogartz \& Wackwitz, $1970,1971)$ and the state of theory development in the vigilance literature, specific predictions regarding interaction effects are probably premature at this time. However, on the basis of previous research concerned with the effects of instruction upon vigilance in which coaction was involved as a static variable (Lucaccini et al., 1968; Tolin, 1970), an Instructions by Coaction interaction was expected. And, on the basis of the results obtained by Hardesty et al. (1963), a Coaction by KR interaction was not expected.

\section{Subjects}

\section{METHOD}

Sixty-four students in general psychology, 32 males and 32 females, served as subjects.

\section{Apparatus \\ The apparatus was a $.91 \mathrm{~m}$ high $\times 4.57 \mathrm{~m}$ long panel painted flat gray, on which three red lights $(2.54-\mathrm{cm}$ diam) were cen- trally situated in a horizontal row at eye level. The middle light served as the signal and the others as nonsignals. The outside lights were each placed $10.16 \mathrm{~cm}$ from the middle light. On top of the signal panel was a second panel, $30.48 \mathrm{~cm}$ high $\times 45.72 \mathrm{~cm}$ long, consisting of a row of lights arranged horizontally. These lights were green, red, and amber, were each $1.27 \mathrm{~cm}$ in diameter, and were used to present KR regarding hits, misses, and false alarms (responses to nonsignals), respectively. This KR panel was lowered out of sight during the no-KR condition. \\ The onset and offset of the signal and nonsignal lights were controlled from an adjoining room by three Hunter Klockounters, and the KR lights were controlled manually by a three-way breaker switch, powered by a 6-V battery. All observers made their responses by depressing a single-channel breaker switch that illuminated a small light in a control room, indicating that a response had been made.}

\section{Procedure}

The subjects were randomly assigned to groups with the restriction that there be four males and four females in each of the eight conditions formed by the factorial combination of the three independent variables, instructions, coaction, and KR.

Each observer was tested individually. The two sets of instructions differed in the following manner. Observers given test instructions were initially told, "This test measures your ability to detect signals which will occur on the panel in front of you." Observers given task instructions were told, "This task simply involves detecting signals which will occur on the panel in front of you."

All observers then received an explanation of the experimental task. If $\mathrm{KR}$ was to be presented, the observer was told how this would occur. Finally, the observer, if wearing a watch, was asked to remove it, and then, if necessary, to ask any questions needed to clarify the task requirements. For the experimenter-present condition, the experimenter sat behind and to the right of the observer.

The vigil lasted $48 \mathrm{~min}$. The observers were not advised of the length of the watch, although they probably knew that it would not exceed $1 \mathrm{~h}$, since they received only one experimental credit for their participation toward their class requirements. The signals occurred at a rate of 3 signals/12 min, with a total of 12 signals occurring during the vigil. The signals were presented at the same time within each 12-min period for all observers, but did not occur at the same time within each 12 -min period. The two nonsignals occurred randomly three times during each 12-min period.

The on-time of the signal and nonsignals was $1.0 \mathrm{sec}$. A miss was recorded if the observer failed to respond within $2.5 \mathrm{sec}$ after the offset of the signal light. KR was provided by the appropriate KR light within $5 \mathrm{sec}$ after the offset of a signal or nonsignal.

\section{RESULTS AND DISCUSSION}

The .05 level of significance was used for all statistical tests. No analysis was made of commission errors, since a total of only six such responses occurred. A factorial analysis of variance was conducted on the percentage of signals detected; between-subjects variables included instructions, coaction, KR, and sex, while time (four 12-min periods) was a within-subjects variable. These percentages and standard deviations are presented in Table 1 for each of the six groups formed from the two levels of each of the three manipulated independent variables.

The three main effects of interest, instructions, coaction, and KR, were, as expected, significant $[\mathrm{Fs}(1,48$ in each case $)=94.11,13.23$, and 36.76, respectively]. The magnitudes of these effects can be seen in the far right-hand column of Table 1 . However, interpretation of these main effects must be qualified by the fact that four significant interactions, all first order, involving these variables were obtained. Two of these interactions involved between-subjects comparisons, while the other two involved within-subjects comparisons. The main effect of sex, as well as all interactions involving sex, was nonsignificant.

With respect to between-subjects interactions, the Coaction by KR interaction, consistent with expectations based on results obtained by Hardesty et al. (1963), was not reliable $(F<1)$. Two other interactions were significant. As predicted, one of these was the Instructions by Coaction interaction $[\mathrm{F}(1,48)=5.82]$. However, the nature of the obtained interaction was not that hypothesized on the basis of the earlier findings of Lucaccini et al. (1968) and of Tolin (1970). It was predicted that an instructions effect would be obtained only if the experimenter was present. While the test- 
Table 1

Mean Percent Signals Detected as a Function of Time on Watch

\begin{tabular}{llrrrrrrrrrr}
\hline & & \multicolumn{10}{c}{ Time Periods (in Minutes) } \\
\cline { 3 - 10 } Condition & Group & Mean & SD & Mean & SD & Mean & SD & Mean & SD & Mean & SD \\
\hline \multirow{2}{*}{ Instructions } & Test & 100.0 & .0 & 100.0 & .0 & 92.0 & .6 & 92.0 & .8 & 96.3 & .7 \\
& Task & 93.0 & .5 & 95.0 & .5 & 71.0 & 1.0 & 63.0 & .8 & 79.7 & 1.5 \\
Coaction & Present & 97.9 & .2 & 97.9 & .2 & 89.6 & .7 & 77.1 & 1.0 & 91.1 & 1.2 \\
& Absent & 93.8 & .5 & 95.8 & .4 & 73.9 & 1.0 & 78.1 & .8 & 84.9 & 1.7 \\
KR & Present & 97.9 & .2 & 97.9 & .2 & 90.6 & .7 & 86.4 & .7 & 93.2 & 1.0 \\
Overall & Absent & 93.8 & .5 & 95.8 & .4 & 72.9 & 1.0 & 68.8 & 1.2 & 82.8 & 1.7 \\
& & 95.8 & .4 & 96.8 & .3 & 81.8 & .9 & 77.6 & .9 & \\
\hline
\end{tabular}

task difference was significant $[\mathrm{t}(30)=5.00]$ in the experimenter-present condition $(94.4 \%$ vs. $84.9 \%$, respectively), the test-task difference was numerically greater $(95.3 \%$ vs. $74.4 \%)$ in the experimenter-absent condition $[\mathrm{t}(30)=6.75]$.

Two possible reasons for the unexpected direction of the interaction are as follows. First, the discrepant findings between the Lucaccini et al. (1968) and Tolin (1970) studies, which lead to the present prediction of an Instructions by Coaction interaction, may have been due to other differences between these two studies rather than coaction. For example, Lucaccini et al. used a complex task designed to simulate vigilance requirements in military-industrial situations, whereas Tolin used a "simple" task. Second, the effects of instructions that refer to the vigil as either a "test" or a "task" may differ from those that refer to the vigil as either a "challenge" or as "monotonous." Perhaps the term "test," more than the term "challenge," leads observers to believe that their performance is being observed and directly evaluated, even if the experimenter is not physically present.

One final comment regarding this interaction. The fact that the test-task difference was greater in the experimenter-absent than in the experimenter-present condition may have been due to a ceiling effect, since the level of detection was quite high in the latter condition. Perhaps further research should measure other aspects of the observer's response, such as latency (Buck, 1966).

The second significant between-subjects interaction involved instructions and $\operatorname{KR}[\mathrm{F}(1,48)=9.17]$. Observers in each instruction condition who received KR detected significantly more signals than observers who did not receive KR. This difference was numerically greater in the task-instruction condition $[87.5 \%$ vs. $71.8 \%$, respectively, $\mathrm{t}(30)=4.04]$ than in the testinstruction condition $[98.9 \%$ vs. $93.7 \%$, respectively, $t(30)=2.25]$. Since the detection rate was quite high for observers in the latter condition, especially those receiving $K R$, this interaction may have resulted from a ceiling effect.

Regarding within-subjects effects, a reliable decrement in vigilance was obtained $[F(3,144)=19.00]$. As can be seen in Table 1, the percentage of signals detected during the last $24 \mathrm{~min}$ of the watch was lower than that during the first $24 \min (79.7 \%$ vs. $96.3 \%$, respectively), a finding that is consistent with Mackworth's (1950) observations.

Both the instructions and $\mathrm{KR}$ variables interacted with time $[\mathrm{Fs}(3,144$ in each case $)=6.41$ and 3.51 , respectively] (see Table 1). A significant vigilance decrement was obtained for observers given task, but not test, instructions. Vigilance decreased reliably across time for observers in both KR conditions; the decrement was greater for those not receiving KR than for those receiving $\mathrm{KR}$. Coaction did not interact with time; a reliable vigilance decrement occurred for both coaction conditions.

As indicated previously, vigilance decrements are more common in laboratory studies than in studies involving military, industrial, or other "real-life" situations. This finding is thought to involve differences in the observers' motivational levels (Mackworth, 1970; Smith \& Lucaccini, 1969). Of the three variables manipulated in the present study, only instructions was effective in preventing a vigilance decrement. Halcomb and Blackwell (1969) have suggested that any incentive used to induce a motivational state must be relevant to the subject's personal motives. The present testoriented instructions, which emphasized the evaluative nature of the vigil, should have been relevant for the observers, since they were college students accustomed to being assessed in their various classes. The effects of the coaction and KR variables might have been greater if the individual present with the observer had been his psychology instructor and if the KR, rather than being presented mechanically, had been presented by the instructor.

\section{REFERENCES}

Antonelli, D. C., \& Karras, G. C. Performance on a vigilance task under conditions of true and false knowledge of results. Perceptual and Motor Skills, 1967, 25, 129-138.

BERGUM, B. O., \& LEHR, D. J. Vigilance performance as a function of paired monitoring. Journal of Applied Psychology, 1962, 46, 341-343. 
BERGUM, B. O., \& LEHR, D. J. Effects of authoritarianism on vigilance performance. Journal of Applied Psychology, 1963, 47, 75-77.

Bogartz, R. S. On the meaning of statistical interactions. Journal of Experimental Child Psychology, 1976, 22, 178-183.

BogarTz, R. S., \& WACKwITz, J. H. Transforming response measures to remove interactions or other sources of variance. Psychonomic Science, 1970, 19, 87-89.

Bogartz, R. S., \& WACKWITz, J. H. Polynomial response scaling and functional measurement. Journal of Mathematical Psychology, 1971, 8, 418-443.

Buck, L. Reaction time as a measure of perceptual vigilance. Psychological Bulletin, 1966, 65, 291-308.

Caron, R. F., \& Caron, A. J. Motivation as a determinant of retroactive inhibition. Journal of Verbal Learning and Verbal Behavior, 1965, 4, 17-24.

Halcomb, C. G., \& Blackwell, P. Motivation and the human monitor: The effect of contingent credit. Perceptual and Motor Skills, 1969, 28, 623-629.

HaRdesty, D., Trumbo, D., \& Bevan, W. Influence of knowledge of results on performance in a monitoring task. Perceptual and Motor Skills, 1963, 16, 629-634.

Huntermark, J. M. Pre-task instructions, coaction, and feedback as motivational variables in human vigilance. (Doctoral dissertation, University of Arkansas, 1977.) Dissertation Abstracts International, 1977, 38, 2399-B. (University Microfilms No. 77-23, 367).

Johnson, E. H., \& PaYne, M. C. Vigilance: Effects of frequency of knowledge of results. Journal of Applied Psychology, 1966, 50, 33-34.

Lucaccini, L. F., Freedy, A., \& Lyman, J. Motivational factors in vigilance: The effect of instructions on perfor- mance in a complex vigilance task. Perceptual and Motor Skills, 1968, 26, 783-786.

MACKWORTH, J. F. Vigilance and attention. Harmondsworth, England: Penguin, 1970.

MACKWORTH, N. H. Researches on the measurement of human performance. Medical Research Council Special Report, No. 268. London: Her Majesty's Stationery Office, 1950.

McCormick, P. D. Performance in a vigilance task as a function of length of inter-stimulus interval. Canadian Journal of Psychology, 1959, 13, 68-71.

McCormick, P. D., Binding, F. R. S., \& McElerhan, W. G. Effects on reaction time of partial knowledge of results on performance. Perceptual and Motor Skills, 1963, 17, 279-281.

Smith, R. L., \& Lucaccini, L. F. Vigilance research: Its application to industrial problems. Human Factors, 1969, 11, 149-156.

ToliN, P. Instruction effects on watch keeping in a "simple" vigilance task. Perception \& Psychophysics, 1970, 9, 227-228.

WiEnER, E. L. Knowledge of results and signal rate in monitoring: A transfer of training approach. Journal of Applied Psychology, 1963, 47, 214-222.

WitTe, K. L., \& Huntermark, J. The effects of instructions, evaluative feedback and knowledge of results upon the short-term retention of ninth graders. Bulletin of the Psychonomic Society, 1975, 5, 79-81.

(Received for publication July $11,1978$. ) 